\title{
AN ASSESSMENT OF THE SPATIAL VARIATION OF ISOTOPIC RATIOS IN A CANDU REACTOR FOR NUCLEAR TREATY MONITORING
}

\author{
Aaron W. Burkhardt, James E. Bevins, Stephen H. Baxter \\ Air Force Institute of Technology \\ 2950 Hobson Way, Wright-Patterson AFB, OH 45433
}

Aaron.Burkhardt@afit.edu, James.Bevins@afit.edu, Stephen.Baxter@afit.edu

\begin{abstract}
A 3-D Quarter-Core CANDU-6 is modeled using Serpent 2 for nuclear treaty monitoring. The spatial variation of flux spectra and isotopic concentrations is analyzed to determine the potential isotopic distribution of key radionuclides from standard reactor operations relevant to non-proliferation. The initial results of the model show a $46 \%$ difference in overall flux magnitude throughout the core as well as a 2-30\% difference in discrete energy flux. The coupled production rate (magnitude) and spectral differences can contribute to significant spatial variations in isotope ratios throughout the core. Initial results indicate ${ }^{239} \mathrm{Pu} /{ }^{240} \mathrm{Pu}$ ratios vary by as much as $51 \%$ across a single CANDU-6 fuel bundle at final burnup. The model is currently being used to develop an accurate representation of spent fuel to perform spatial isotopic analysis across the entire CANDU-6 core.
\end{abstract}

KEYWORDS: CANDU, Isotopic Variance, Monte-Carlo Transport, Non-Proliferation, Nuclear Forensics

\section{INTRODUCTION}

The Comprehensive Nuclear Test Ban Treaty Organization (CTBTO) maintains the International Monitoring System (IMS) to detect proliferant activities and limit the proliferation of nuclear weapons. The IMS network covers 337 locations around the world with a variety of sensors including seismic, waveform, and radionuclide detectors [3]. Of interest to this work, eighty of these stations have radionuclide sensors for measuring traces of radioactive isotopes in collected air samples. The measured isotopic ratios can be used to inform their origin, such as weapons testing, fuel reprocessing, reactor operations, or one of several other nuclear activities as some isotopic ratios are sensitive to the creation environment. The CTBTO developed a Standard Recommended List of radioactive isotopes to prioritize isotopes of importance to non-proliferation [1-3].

To determine if proliferation is taking place, the results of IMS stations are compared to either a recorded database of signatures and/or models[3]. Current models of reactor operations for nonproliferation and radioisotope release generally neglect key parameters that can affect the range of isotopic signatures seen possibly affecting the resulting activity categorization. For example, possible assumptions include: no axial variation, core averaged isotopics, or even infinite systems. The goal of this research is to explore the potential range of isotopic ratios and test the validity of these assumptions. 
This work uses Serpent 2 to develop a Quarter-Core Canada Deuterium Uranium (CANDU-6) reactor model and perform an analysis on spatial variation of neutron flux spectra and isotopics from burnup [4]. Sec. 2 describes the geometry, materials, model physics, and assumptions used for the Serpent 2 CANDU-6 model. Sec. 3 is broken into three parts. The first part analyzes the spatial variation of the neutron flux spectra from a criticality model to determine the overall magnitude range as well as differences in neutron energy. The second part depletes the initial model, mimicking standard CANDU-6 refueling practices, to obtain an accurate representation of spent fuel to determine the spatial variation of key isotopic ratios. Last, an initial analysis of a single bundle is performed to demonstrate the methodology and spatial variation.

\section{MODEL DESCRIPTION}

The CANDU model is based on the stylized half-core model described by Pounders [5]. For this study, a quarter-core model, shown in Fig. 1, is developed from the right half of the half-core Pounders model. A quarter-core model is used to improve computational efficiency by leveraging core symmetry. The Pounders model was modified to a general CANDU-6 design by including the geometry of the heavy water reflector and modifying the isotopics of the materials used $[5,6]$.

\subsection{Geometry and Materials}

The quarter-core model contains 1140 CANDU-6 fuel bundles arranged in 95 channels of 12 bundles each. The fuel bundle has a square pitch of $28.575 \mathrm{~cm}$ and a length of $49.53 \mathrm{~cm}$. Each bundle consists of 37 naturally enriched $\mathrm{UO}_{2}$ fuel pins $0.6103 \mathrm{~cm}$ in radius with $0.0419 \mathrm{~cm}$ thick zirconium cladding surrounded by heavy water coolant. These are arranged in three concentric rings around a center pin. A zirconium pressure tube and calandria tube surround the pin lattice, and heavy water with trace amounts of boron from the startup process fill in the rest of the lattice pitch $[5,6]$.

The 95 fuel channels are $594.36 \mathrm{~cm}$ in length and are arranged in an $11 \times 11$ quarter-cylindrical shape as shown in Fig. 1. They are surrounded by an average of $65 \mathrm{~cm}$ of heavy water used as a reflector and $41.9 \mathrm{~cm}$ of calandria tube shielding in the radial direction. The space outside of the calandria tube is considered void, and no additional moderation or shielding is added in the axial direction $[5,6]$.

Three rows of three and a half AISI type 304 stainless steel (SS304) adjuster rods were located at $217.49,297.18$, and $377.18 \mathrm{~cm}$ from the axial end of the core and inserted $171.45 \mathrm{~cm}$ into the quarter-core model as weak neutron absorbers as shown in Fig. 1. Each adjuster rod is split into two regions, consisting of a small SS304 pin, a SS304 tube, and a zirconium guide tube. The upper region of each rod extends $85.725 \mathrm{~cm}$ from the boundary, and the lower region continues an additional $85.725 \mathrm{~cm}$ into the core. The dimensions for the pin and tubes in each region are shown in Table 1. Heavy water fills gaps between each region.

Finally, the planes corresponding to 270 and 0 azimuthal degrees from the origin of the core were set as reflective boundaries to mimic the symmetry of a full-core model. The remaining planes in the $-y,+x$, and both axial directions were set as vacuum boundaries to prevent reflection back into the system. This vacuum boundary assumption slightly underestimates the neutron reflection in 


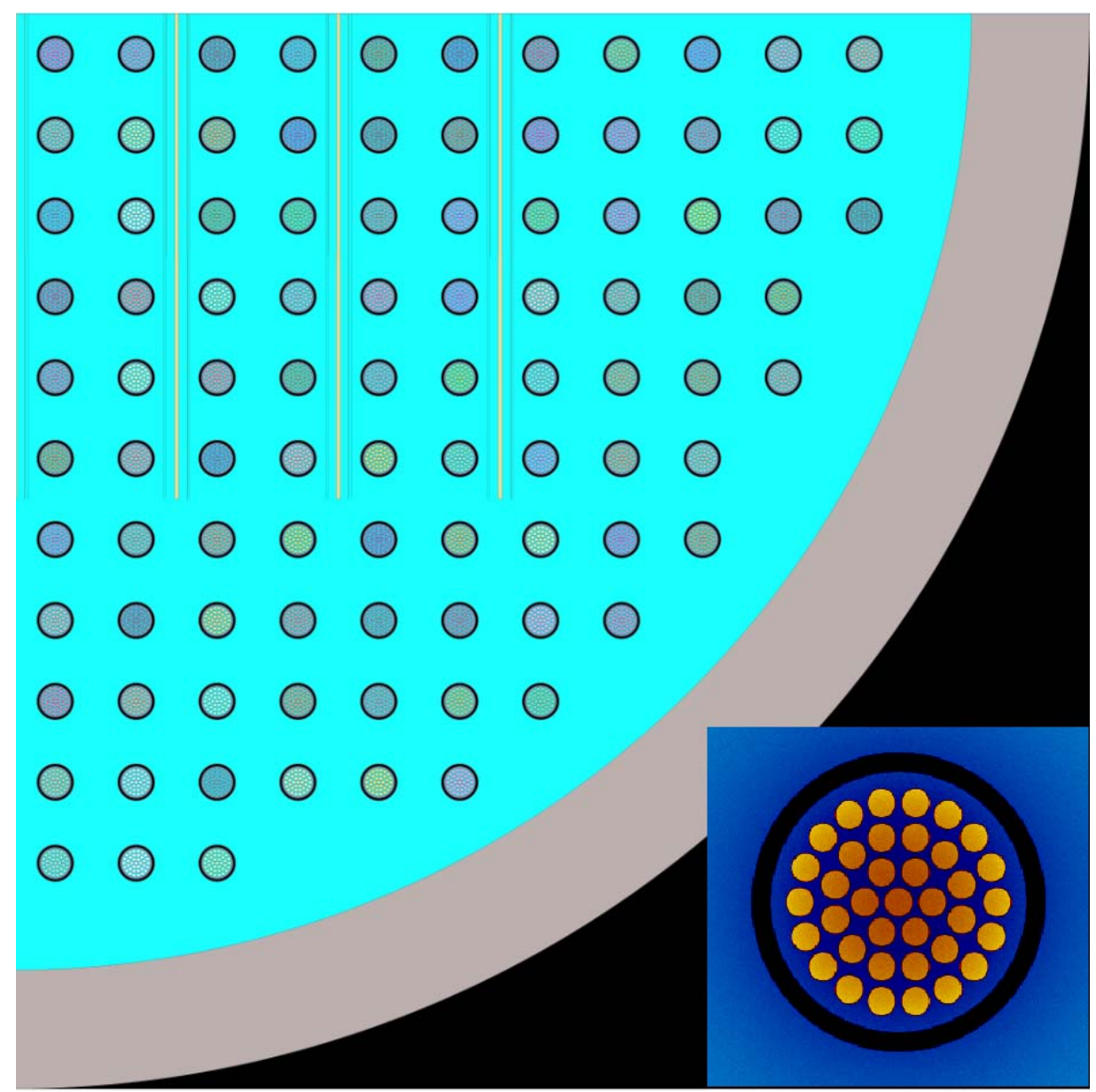

Figure 1: Illustration of the Quarter-Core CANDU-6 Model Depicting Core Geometry and Bundle Geometry from an Axial Slice

Table 1: Adjuster Rod Regional Dimensions [5]

\begin{tabular}{|c|c|c|}
\hline & Upper Region $(\mathrm{cm})$ & Lower Region $(\mathrm{cm})$ \\
\hline Shim OR & 0.650 & 0.710 \\
\hline Steel Tube IR & 3.607 & 3.607 \\
\hline Steel Tube OR & 3.725 & 3.690 \\
\hline Guide Tube IR & 4.519 & 4.519 \\
\hline Guide Tube OR & 4.572 & 4.572 \\
\hline
\end{tabular}

the axial directions. This reflection is considered negligible for this work, an assumption that is consistent with literature $[5,6]$. 


\subsection{Model Physics}

\subsubsection{Fuel burn correction}

If the model consisted entirely of fresh fuel, it would be significantly supercritical with a $\mathrm{k}_{\infty}$ of $\sim 1.122$ [6]. This is offset with burnable poisons that are not required during the steady-state operation of the reactor. Therefore, each bundle was artificially burned to account for the buildup of fission products, to deplete the fuel, and to create a power profile more characteristic of steadystate CANDU-6 reactor operations.

A single fuel bundle with periodic boundary conditions, simulating an infinite system, was modeled in Serpent 2 and depleted to eight burnup targets shown in Table 2 [4,5]. In Serpent's burnup mode, the Linear-Extrapolation/Quadratic-Interpolation Predictor-Corrector (LE/QI) scheme was used as well as a Chebyshev Rational Approximation Method (CRAM) order of 16 for each burnup step [7,8]. LE/QI and CRAM are respectively higher order and sub-step methods used to solve the Bateman equations for increased accuracy in isotopic concentrations while reducing the required number of burnup steps. Four additional burnup sub-steps were added between each burn-up target to ensure the convergence of the depleted materials. Each transport cycle consisted of 1,000 active generations and 100 skipped generations of 10,000 neutrons each. A power density of 21.34 MW/MTU was used, and all isotopic data of the fuel were recorded. The isotopics from each burned target were incorporated into the quarter-core model in accordance with Pounders [5]. This artificial fuel burn is successful in obtaining a general power profile; however, further power profile smoothing is discussed in Sec. 3.

Table 2: Burnup Targets for Fuel Burn Correction [5]

\begin{tabular}{|c|c|}
\hline Target & Burnup (MWd/MTU) \\
\hline 1 & 32.69 \\
\hline 2 & 78.38 \\
\hline 3 & 342.37 \\
\hline 4 & 818.87 \\
\hline 5 & 1638.73 \\
\hline 6 & 3608.15 \\
\hline 7 & 6381.44 \\
\hline 8 & 8721.49 \\
\hline
\end{tabular}

\subsubsection{Data and optimization}

Continuous ENDF/B-VII.1 cross-section data were used for the unionized energy grid [9]. ZeroKelvin cross-section data were used for Doppler-Broadening Rejection Corrections on the uranium 
and plutonium isotopes to account for the impact of thermal motion on angular distributions of elastic scattering near resonances [10]. Unresolved resonance probability table sampling was used on isotopes where the resonance peaks overlap in the high resonance energy region to accurately account for self-shielding [10]. Additional thermal scattering libraries from ENDF/B-VII.1 were used for the hydrogen and deuterium in the water. The tolerance for the unionized energy grid was set to $5 \mathrm{E}-5$ to reduce the required memory. This is the same value that is set as default for burnup calculations and is considered to have negligible effects on results [10].

\subsubsection{Criticality Model Run conditions}

The total fuel mass of the quarter-core model is approximately 25.4 MTU. The model was run at a 21.34 MWt/MTU power density. A fission heat deposition detector was used to measure the power in each channel and produce a power profile across the quarter-core model. The most bounding bundles, AA7 and KA1, were selected for flux spectrum analysis. The flux detector measured the neutron spectrum inside of the fuel material using 500 equal lethargy bins. Finally, the transport cycle used 2,000 active generations and 250 inactive generations of 200,000 neutrons each to obtain a low uncertainty for these detectors.

\subsubsection{Burnup Model Run conditions}

The quarter-core criticality model was used as the basis for the burnup model. The model was burned for three burnup steps to 2.8 Full-Power Days (FPD) at a time. Then, one channel was selected for refueling via the Swing-Eight Refueling Scheme [11]. Each transport cycle simulated 40 inactive and 1000 active generations of 100,000 neutrons each. Channels were selected for refueling based on their total channel burnup (target $7.5 \mathrm{GWd}$ ). Their burnup with respect to other channels was also taken into consideration to maintain criticality and an even power distribution.

\section{RESULTS}

\subsection{Criticality Model}

The results of the initial criticality model show a coarse power profile ranging from $2.08 \mathrm{MW}$ to $7.77 \mathrm{MW}$, shown in Fig. 2, with a $\mathrm{k}_{\text {eff }}$ of $1.00244 \pm 2.2 \mathrm{E}-5$. This was expected as eight target burnups for the entire distribution of the core is too coarse for final analysis; however, this provides intermittent answers to the potential scale of spatial variance within the model. The average channel power for a CANDU-6 reactor is 6.6 MW for the inner core and 3.0 MW for the outer core [6]. This initial model shows an uneven power distribution, which is addressed in Sec. 3.2 where a smoother power profile is obtained after 60 channel refuelings.

A comparison of the normalized flux spectra, with one sigma uncertainty, from the hottest and coldest bundles, AA7 and KA1, is shown in Fig. 3. The overall magnitude difference of the two bundles is $46.08 \pm 4.6 \% \mathrm{~cm}^{-2} \mathrm{~s}^{-1}$. To compensate for the vast difference in flux magnitude, the two spectra are normalized by their peak flux to show differences in the overall energy-dependent flux spectra. The resonance region has several differences ranging from 2-5\% difference. The upper edge of the thermal peak shows one of the largest differences at $\sim 8 \%$. This is expected to contribute to differences in the production rate of thermal activation isotopes. The largest difference is the 
Initial

A $\quad B \quad C \quad D \quad E \quad F \quad G \quad H \quad$ I J $K$

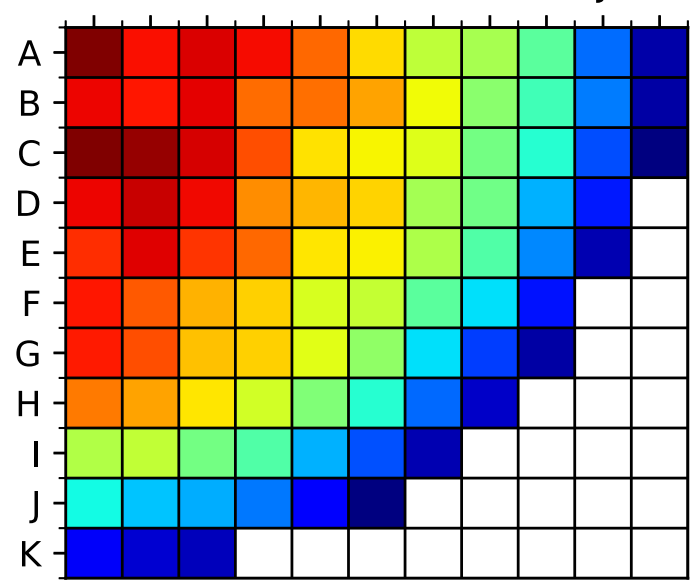

Refuel \#60

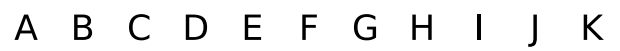
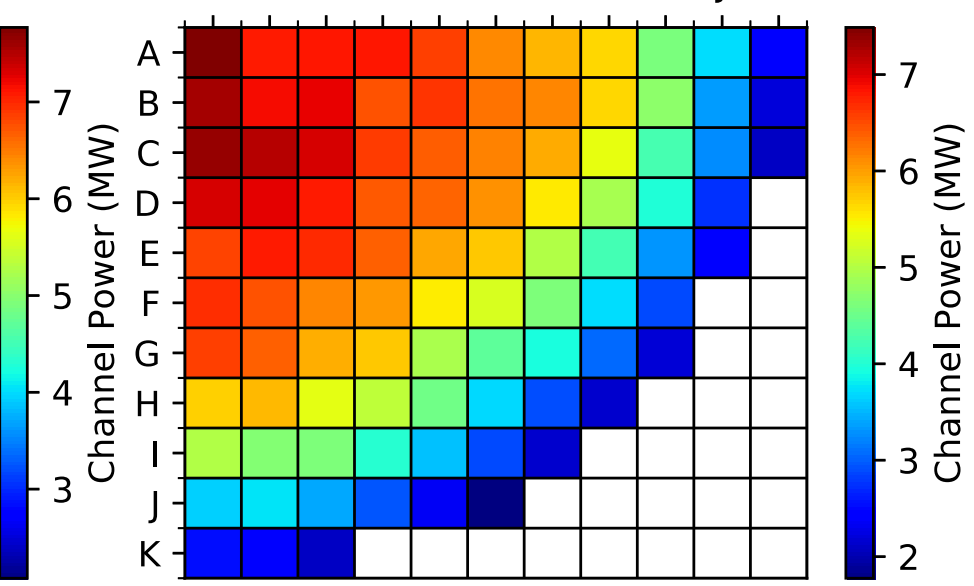

\section{Figure 2: Power Profile Generated from Integrated Channel Powers for the Initial Burn Loading in Table 2 (left) and after 60 Channel Refuelings (right) Illustrating the Profile Smoothing Achieved from Refueling}

first resonance peak with a value of $30 \%$. These differences are primarily due to differences in burn-up and burn-up rates in the two bundles.

\subsection{Burnup Model}

Following the burnup run conditions described in Sec. 2, the initial power profile of the quartercore CANDU model has been smoothed as shown in Fig. 2. Each refueling contributes to the overall accuracy of the model by creating a more realistic burnup distribution and more closely approximates steady state conditions. This is essential in accurately creating the flux spectrum in each bundle used for the depletion calculations.

This research on the spatial variation of isotopics using this model is currently ongoing. With each refueling of the quarter-core model, injected uncertainty from the artificial burnup is removed. The results of this burnup process, including the spatial isotope ratio distribution, will be presented at the Physor 2020 Conference.

\subsection{Single Bundle Analysis}

A single bundle with periodic boundary conditions was also analyzed to obtain preliminary results and determine the spatial variation of isotopics within a bundle. The results, shown in Fig. 4, indicate a difference of $51.1 \%$ in the ${ }^{239} \mathrm{Pu} /{ }^{240} \mathrm{Pu}$ between the inner ring and the outer ring at final burnup. The absolute values of these ratios at final burnup ranged from 1.37 to 2.07. No significant differences were observed axially within a fuel pin. 


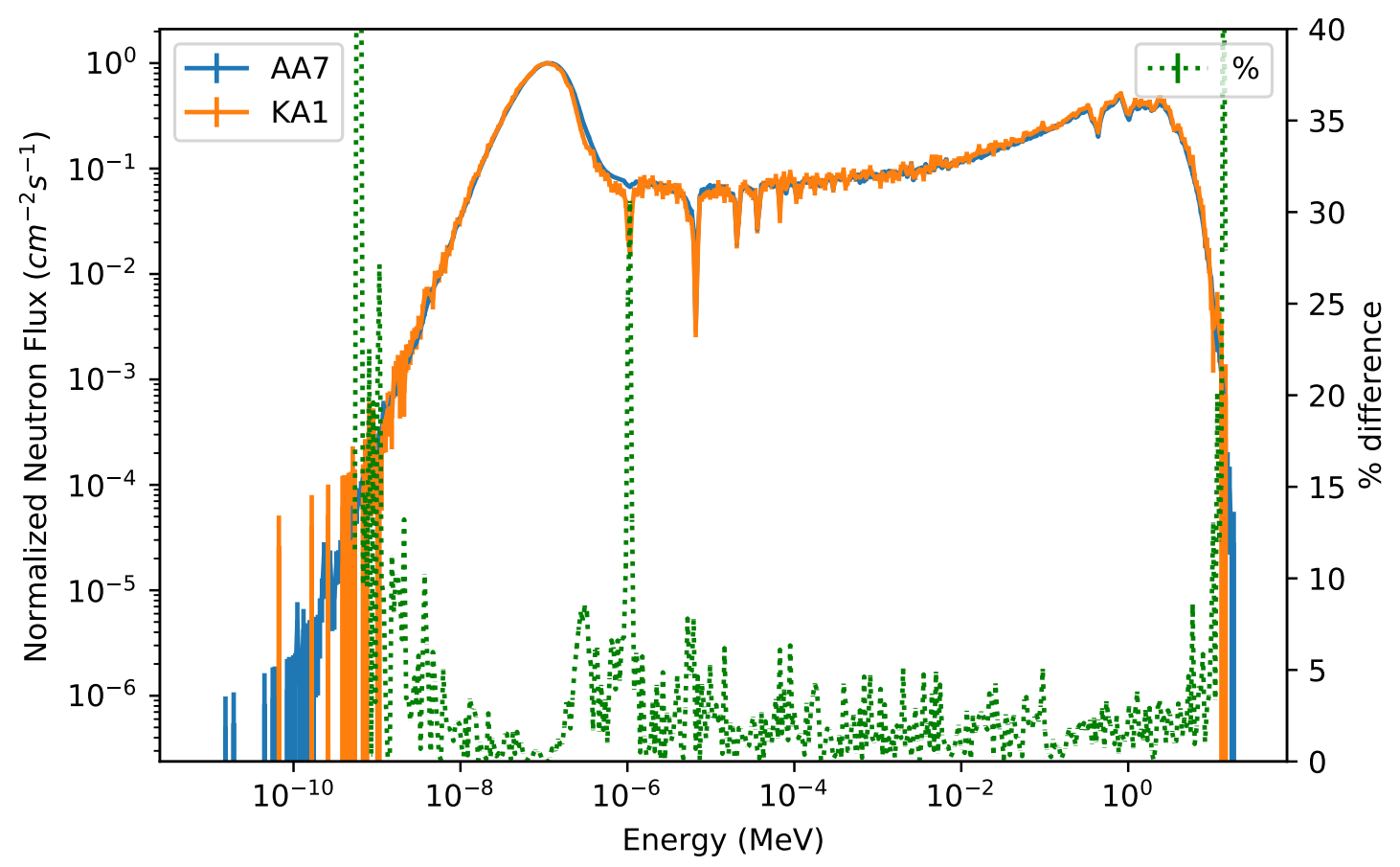

Figure 3: Comparison of Normalized Neutron Flux Spectra for Bundles AA7 and KA1 Highlighting the Differences in Discrete Neutron Energy

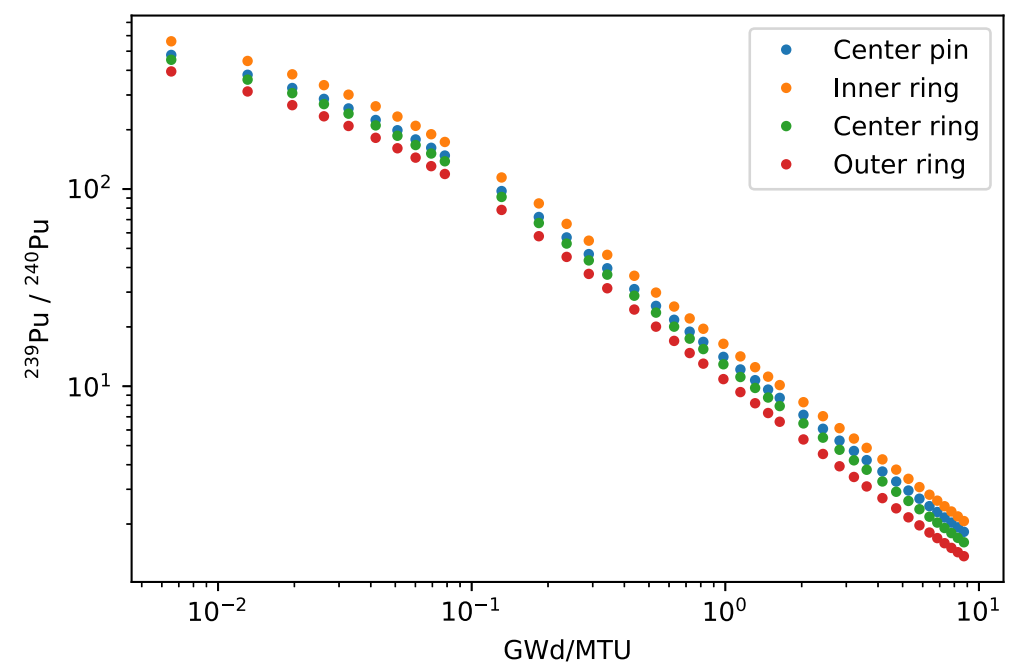

Figure 4: Single Bundle Burnup Analysis of ${ }^{239} \mathbf{P u} /{ }^{240} \mathbf{P u}$ 


\section{CONCLUSIONS}

From preliminary assessment of the criticality model, burnup model, and single bundle analysis, the presence of isotopic spatial variance is significant. The $51.1 \%$ variation in ${ }^{239} \mathrm{Pu} /{ }^{240} \mathrm{Pu}$ within a bundle, coupled with the $46 \%$ flux magnitude differential and 2-30\% discrete energy differences, provides the expectation for large isotopic ratio differences not only between the center and peripheral of the core but between neighboring channels and bundles. This variation carries potentially important implications for monitoring radio-isotope signatures at IMS stations, estimating plutonium production, and quantifying proliferant activities. The complete assessment of the quarter-core model along with the full isotopic distribution of the quarter-core model on a bundle basis will be available and presented at the Physor 2020 Conference.

\section{ACKNOWLEDGEMENTS}

This research was supported in part by the U.S. Air Force Technical Application Center (AFTAC) under the AFIT/AFTAC Endowed Term Chair MOA\#: 212196. The authors would also like to thank Dr. Darren Holland, Dr. Abigail Bickley, Dr. Jaakko Leppänen, Dr. Rob Smith, and Dr. Jeremey Kephart for their help in this work.

\section{REFERENCES}

[1] J. Medalia, "Comprehensive Nuclear-Test-Ban Treaty: Background and Current Developments," CRS Report for Congress, (2008).

[2] T. Ansaranta, et al., "Comparison of Radionuclide Data Analysis Results of the CTBTO/IDC and the Finnish NDC," Helsinki University of Technology, (2001).

[3] "The International Monitoring System," CTBTO Annual Report, (2017).

[4] J. Leppänen et al., "The Serpent Monte Carlo code: Status, development and applications in 2013," Annals of Nuclear Energy, 82, pp. 142-150 (2015).

[5] J.M. Pounders et al., "A 3D stylized half-core CANDU benchmark problem," Annals of Nuclear Energy, 38, pp. 876-896 (2011).

[6] B. Rouben, "Reactor-Physics Analysis Basis for Current CANDU," Presentation to US $N R C$, https://www.nrc.gov/docs/ML0300/ML030020086.pdf, (2002).

[7] A.E. Isotalo, P.A. Aarnio, "Higher order methods for burnup calculations with Bateman solutions," Annals of Nuclear Energy, 38, pp. 1987-1995 (2011).

[8] A.E. Isotalo, P.A. Aarnio, "Substep methods for burnup calculations with Bateman solutions," Annals of Nuclear Energy, 38, pp. 2509-2514 (2011).

[9] M.B. Chadwick et al., "ENDF/B-VII.1 Nuclear Data for Science and Technology: Cross Sections, Covariances, Fission Product Yields and Decay Data," Nuclear Data Sheets, 112, pp. 2887-2996 (2011).

[10] J. Leppänen, "Serpent - a Continuous-energy Monte Carlo Reactor Physics Burnup Calculation Code," (2015)

[11] B. Rouben, "CANDU Fuel-Management Course," (2002). 